2019-08-28

Developing a coupled turbine thrust methodology for floating tidal stream concepts: Verification under prescribed motion

\author{
Brown, Scott Andrew
}

http://hdl.handle.net/10026.1/14879

10.1016/j.renene.2019.08.119

Renewable Energy

Elsevier

All content in PEARL is protected by copyright law. Author manuscripts are made available in accordance with publisher policies. Please cite only the published version using the details provided on the item record or document. In the absence of an open licence (e.g. Creative Commons), permissions for further reuse of content should be sought from the publisher or author. 


\title{
Developing a coupled turbine thrust methodology for floating tidal stream concepts: Verification under prescribed motion
}

\author{
S.A. Brown ${ }^{\mathrm{a}, *}$, E.J. Ransley ${ }^{\mathrm{a}}$, D.M. Greaves ${ }^{\mathrm{a}}$ \\ ${ }^{a}$ School of Engineering, Computing and Mathematics, University of Plymouth, Plymouth, \\ United Kingdom, PL48AA
}

\begin{abstract}
Floating systems offer an opportunity to expand tidal energy resource through an increase in viable sites and greater flow speeds near the free surface. However, the close proximity of the free surface provides uncertainty regarding power delivery and survivability due to the presence of waves, which could be addressed through a numerical model that is capable of considering all components of a floating tidal system simultaneously. This paper presents the first step in the development of such a tool: using the open-source CFD libraries of OpenFOAM as a basis, a computationally efficient HATT model has been developed for generalised incident flow conditions using actuator theory. A thorough evaluation of the model's sensitivity to key considerations in the simulation of entire floating tidal systems, such as flow speed and mesh alignment, showed that the model is robust, ensuring that it is suitable for future extension to wave-driven environments and integration into a framework for such systems.
\end{abstract}

Keywords:

HATT model, CFD, OpenFOAM, Actuator theory, Marine renewable energy, Velocity deficit

\section{Introduction}

Development of the Offshore Renewable Energy (ORE) sector is of high national importance for the UK and tidal stream represents a renewable energy source with a number of desirable characteristics: it is more predictable than other sources (such as wind and wave energy) providing simplified power grid management; the resource tends to be concentrated by topography resulting in desirable sites with high energy densities close to land masses (and to end users), reducing costs in terms of installation and maintenance as well as cabling, and; the majority of present device concepts, particularly Horizontal Axis Tidal

\footnotetext{
* Corresponding author

Email address: scott.brown@plymouth.ac.uk (S.A. Brown)
} 
Turbines (HATTs), benefit from technology that has been developed through existing industries, such as hydro and wind power, accelerating the maturity of the tidal stream industry.

However, the majority of the proposed tidal stream devices (particularly those with the highest level of technology readiness) tend to be seabed-mounted or gravity-based devices. Use of these concepts limits the number of viable sites as the water depth has to fall within a narrow range, due to constraints on the installation and the required clearance above the turbine blades. The bathymetry also has to be favourable, i.e. relatively flat and horizontal. Furthermore, due to boundary layer effects, the flow speed at depth tends to be lower and so seabed mounted devices do not exploit the full tidal stream resource at deeper sites, providing a further constraint on water depth. Finally, seabed-mounted devices tend to suffer from time-consuming and difficult installation, maintenance and recovery procedures, greatly increasing the overall cost of the projects.

Consequently, a number of floating tidal stream concepts have been proposed. These concepts have a number of distinct advantages over seabedmounted devices. Floating devices are not limited by water depth, bathymetry or the presence of mobile sediments resulting in a greater number of potential sites and hence a higher potential extractable resource. In addition to this, floating tidal stream concepts tend to be easier and quicker to install, maintain and recover since the majority of them can be towed to site using basic tug boats, reducing the need for expensive specialist vessels. Floating devices also benefit from positioning the turbine towards the top of the water column where the flow velocity is maximum, again increasing the available resource compared with seabed-mounted devices at certain sites.

However, despite the advantages of floating tidal stream concepts, it should be noted that sites ideally suited for bottom mounted turbines tend to be shallower, and hence generally experience faster flow speeds than deeper sites. Furthermore, the flow observed by bottom-mounted and floating designs would be similar at these shallower sites since the location of the turbine will be closer to mid-water in both cases, limiting the advantage of a floating approach. Floating devices also require additional considerations to be made, regarding their location at the free-surface: firstly, these devices have an additional impact on other stakeholders at the site, e.g. obstruction of navigation and visibility issues, and; secondly, these devices are exposed to free-surface effects and waves leading to concerns over both the power delivery and the survivability of the devices. With so few deployments and limited operating hours to date, the effect of proximity to the free-surface and wave-induced motion/loading on these devices is not presently understood and crucial, under-pinning research is required before these devices will become commercially viable.

As with other emerging ORE industries, modelling (both physical and numerical) has now become an essential part of the development process. Numerical modelling, in particular, is being relied upon more and more to overcome the costs and scaling issues associated with physical modelling as well as to provide the high resolution measurements and the quantitative descriptions required for engineering design. 
The modelling of floating tidal stream concepts, however, is incredibly complex, combining: complicated hydrodynamics, such as the interaction between waves and currents; floating structure; mooring system, and; (possibly multiple) submerged turbines. Existing numerical codes are rarely capable of including all of these elements and, for those that are, the behaviour tends to be linearised and each of the elements treated separately, i.e. a 'decoupled' model. This creates considerable uncertainty in the power delivery and survivability predicted by these models. A model which fully resolves the contribution of all elements simultaneously as well as the fully nonlinear hydrodynamics and floating-body motion is therefore desirable when assessing the behaviour of these devices, the loads (in the mooring lines for example) and the power output from the turbine. Unfortunately, such a model, if available, would likely suffer from excessive CPU requirements making the use of such a tool prohibitive in routine design processes.

Therefore, this paper details the first step in an incremental development of an efficient numerical tool that is capable of predicting the fully nonlinear, coupled behaviour of floating tidal stream systems. The article concentrates on the methodology used to generate a computationally efficient HATT model that predicts accurately the coupled forces on the turbine, and the fluid, while remaining numerically stable under arbitrary motion. Using the open-source Computational Fluid Dynamics (CFD) libraries of OpenFOAM as a basis, the HATT model has been developed for generalised incident flow conditions using actuator theory. It should be noted that actuator approaches have been implemented in OpenFOAM in previous work, but they have largely focused on validation of fixed wind turbine wake predictions [25, 26, 33, 44], and the implications for wind farm layout $[32,49]$. Although these previous methods for fixed turbines provide a basis for floating applications, they are not directly applicable since they often require a very specific mesh layout to maximise alignment with the turbine, which could not be achieved if the turbine position is constantly updating. Therefore, the approach presented here incorporates the effects of the turbine model on the fluid dynamics in the fully nonlinear Reynolds-Averaged Navier-Stokes (RANS) solver via a 'body-force', momentum-sink-type methodology which allows the turbine position to move independently of the mesh. This results in a strongly coupled model that is rigorously characterised, using steady-state simulations, and demonstrated to be robust in a series of test cases in which the turbine has prescribed motion.

\section{Background}

High-fidelity numerical methods, such as CFD, have been used extensively in mature industries, like wind energy, to assess the behaviour and performance of horizontal axis turbines. The development of tidal stream turbines has benefited greatly from the knowledge gained in the wind industry, however; it is important to recognise that tidal turbines can be subject to free-surface effects (such as ventilation), possible cavitation and bi-directional flow and that the established methods must be adapted to provide an accurate prediction of tidal stream 
turbine behaviour [35]. Moreover, existing methods rarely take into account the arbitrary motion of a freely moving turbine which, in the case of a floating tidal stream device, could result in a turbine velocity comparable to the free-stream velocity of the fluid. The following is a brief review of the most commonly used methods for turbine modelling in CFD.

Arguably, the most realistic methods are 'bladed-resolved' techniques, in which the turbine is directly meshed into the computational domain, allowing the flow to be resolved as it passes the turbine blades [15, 19, 21, 43, 50]. Computationally, these methods are extremely expensive; the spatial resolution must be fine enough in the vicinity of the turbine to accurately model both the complex geometry of the turbine and the small-scale flow structures around the blades; and the time step must be shorter than the temporal scales of these small-scale structure, in order to resolve them accurately. Furthermore, the mesh needs to be updated at every time step to accommodate the rotation of the turbine, which often involves complex remeshing techniques (e.g. arbitrary mesh interface (AMI) [46]), or additional interpolation overheads (e.g. overset grid [21, 50]), further reducing the computational efficiency. Thus, although potentially very accurate, the computational cost of blade-resolved methods is often considered to be prohibitive in routine design processes and so cheaper alternatives have been developed, aiming to represent the required characteristics of the turbine without the need to resolve the flow around the turbine blades.

Actuator methods are a common approach when representing a horizontal axis turbine in a fluid flow. The simplest cases are actuator disc models which, based on momentum theory, apply a 'resistance' to the incoming flow over the swept area of the turbine, similar to that of a porous disc (which is often used in physical laboratory experiments as a simple representation of a turbine $[3,22$, 31]). The applied resistance typically takes the form of a momentum sink, the magnitude of which is based on the relationship between the free-stream velocity and the thrust on (and power generated by) the turbine [6]. In these methods a coarser, static mesh can be used, greatly reducing the computational costs relative to blade-resolved methods. A number of authors have utilised actuator disc models, in a wide range of numerical models including CFD simulations of both wind $[2,7,36,45]$ and marine current $[1,4,5,13]$ turbine applications. However, it has often been found that an actuator disc approach suffers from the absence of rotational effects (particularly when the focus is on an accurate prediction of the turbine wake $[13,12,38])$. Since the area of the actuator disc is fixed, vorticity is shed into the wake as a continuous sheet from the edges of the disc instead of from the tips of the blades [38]. To increase the accuracy in unsteady flows and capture rotational effects, extensions to the actuator disc methodology have been developed. These include: actuator line $[8,16,29,42,41]$ and actuator surface $[20,40,47]$ methods in which the applied momentum sink is distributed into finite lines or surfaces to represent the blades of the turbine. Furthermore, in these methods the momentum sink is considered to be transient with the position of the blades being updated based on the flow speed and the characteristics of the turbine. In these methods, the torque on the generator can then be calculated from the angular velocity of the rotation. 
In addition, by discretising the blades in this way, distinct tip vortices can be calculated (rather than the continuous vorticity sheet arising from an actuator disc model) giving an improved representation of the turbine wake [38].

Blade element momentum theory (BEMT) $[4,12,24,27]$ is another extension of the actuator disc model, combining blade-element and actuator methodologies to calculate the lift and drag forces on each section of the discretised turbine blades [6]. With the inclusion of a 'tip loss correction factor' [27, 30, 39], which accounts for vortex effects at the blade tips, BEMT has been shown to have good agreement with physical measurements and blade-resolved CFD models [23, 27, 24]. Masters et al. [27] also suggests that further improvements can be made by including a 'hub loss correction factor' [30] which, in a similar way to the tip loss correction, accounts for vortex effects caused by the presence of the rotor hub.

\section{Methodology}

A new library, allowing for the representation of tidal turbines, has been designed and implemented in OpenFOAM (v. 4.1 [48]), an open source toolbox aimed at solving continuum mechanics problems (including CFD). The software is written in $\mathrm{C}++$ and is based around the Object Orientated Programming (OOP) paradigm, offering a large collection of solvers and shared libraries. Consequently, the new turbine library is easily coupled with many of the existing solvers. However, in this study the focus is on three solvers of increasing complexity, that solve the incompressible RANS equations using the Finite Volume Method (FVM): simpleFOAM, for steady-state simulations; pisoFOAM, for transient single fluid cases; and interFOAM for simulating free surface flows using a two-phase Volume Of Fluid (VOF) approach [37].

Since it is computationally expensive to resolve the flow structure around the turbine, a simpler, more efficient approach for modelling the turbine is adopted (compared with a blade-resolved method). Furthermore, since the overarching aim of this work is to develop a tool for modelling complete floating tidal stream devices, the focus here is on facilitating the key aspects required for a coupled system, i.e. accurate prediction of the coupled forces and numerical stability with arbitrary motion of the turbine, rather than on developing a new turbine model. Therefore, in this study, an actuator disc model has been used to demonstrate the methodology (it is, however, worth noting that, due to the object oriented nature of the code developed here, it is relatively straightforward to include more sophisticated turbine models, such as a BEMT approach, in the future).

To allow for arbitrary movement of the turbine through the computational domain, the turbine model here is based upon a 'weighted body force implementation' which, at each time step, identifies and applies weights to a finite 'region' of the computational domain (representing the turbine). This requires no constraints on the local mesh structure (a requirement for the complete, coupled device), contrary to common methods used in static cases which often require the mesh to be highly contrived in the disc region $[7,12,24]$. These 
weights are then used to determine the local velocity at the turbine position as well as to add an additional, equal and opposite force (based on the thrust on the turbine) to the momentum equations, ensuring that the model is two-way coupled.

\subsection{Actuator Disc Theory}

Actuator disc theory states that, in a steady current, the mass flow rate must be conserved. Hence, the stream-wise velocity at the disc, $u_{t}$, can be determined through the relationship

$$
u_{t}=(1-a) u_{\infty}
$$

where $u_{\infty}$ is the free stream velocity, and $a$ is the axial induction factor [6]. Using momentum theory it is then possible to formulate expressions for the thrust, $T$, on and power, $P$, generated by the disc as functions of the free stream velocity

$$
T=\frac{1}{2} \rho C_{t} A u_{\infty}^{2}
$$

$$
P=\frac{1}{2} \rho C_{p} A u_{\infty}^{3}
$$

where $A$ is the area of the $\operatorname{disc}$ and $C_{t}$ and $C_{p}$ are the thrust and power coefficients respectively, where

$$
C_{t}=4 a(1-a)
$$

$$
C_{p}=4 a(1-a)^{2} .
$$

Actuator disc methods are common in numerical models due to their simplicity, requiring only knowledge of the thrust coefficient and the free-stream velocity. In this study, however, the turbine methodology is required to work in transient flows such as those experienced in wave-driven environments, and hence the free stream velocity is not known a priori. Therefore, the actuator disc methodology is reverse engineered based on the known velocity at the turbine in order to estimate the instantaneous free stream velocity and the thrust on the disc (explained further in Section 3.4).

\subsection{Weighting Function}

The first stage in the turbine model is the calculation of the weighting function (or field), $W$, which determines the contribution of each cell in the computational domain to the local flow velocity at the turbine ( $W$ also determines the distribution of the thrust force on the turbine and the corresponding distributed momentum sink (see Section 3.4)).

In this study, actuator disc theory is used to represent a HATT and so a cylindrical region is selected to represent the turbine. The cylinder has: a radius, $R$, equal to the radius of the swept area of the turbine blades, and; an axis coincident with that of the turbine. At each time step, all cells in the computational domain are evaluated to find the distance between their centre 
and the central line of this 'turbine region', i.e. the turbine axis. For a turbine axis parallel to the global $x$-axis,

$$
\mathbf{d} \mathbf{x}=\mathbf{x}_{c e l l}-\mathbf{x}_{h u b}=(d x, d y, d z),
$$

where $\mathbf{x}_{c e l l}$ and $\mathbf{x}_{h u b}$ are the coordinates of the cell centre and the hub position of the turbine respectively. The $x$-component, $d x$, corresponds to the axial distance from the turbine plane and the axial width of the turbine is $2 N \sigma$ (Figure 1). The radial components of the cylindrical region, $d y$ and $d z$, are used to define another vector

$$
\mathbf{r}=(0, d y, d z),
$$

whose length, $|\mathbf{r}|$, determines the radial distance from the turbine axis. The edge of the turbine will then be located on the line $|\mathbf{r}|=R$. Consequently, the turbine region is made up of cells that have $r$ values that fall within the range $R_{h u b} \leq|r| \leq R$, where $R_{h u b}$ is the hub radius (Figure 1a).

In order to ensure mathematically smooth values for the calculated local flow speed (and thrust forces) through time, for the general case in which the motion of the turbine is not concurrent with the motion of the mesh cells, the turbine region is given a finite width. The width is defined as $2 N \sigma(N \sigma$ either side of the centreline in the axial direction), where $\sigma$ is the Gaussian root mean square width as shown in Figure $1 \mathrm{~b}$ and $N$ is a user-defined coefficient to limit the width of the turbine region (set by default to 2 according to the sensitivity analysis in Section 4.2). A Gaussian weighting, is then determined for each cell in the computational domain

$$
W= \begin{cases}\frac{1}{\sigma \sqrt{2 \pi}} \exp \left(-\frac{d x^{2}}{2 \sigma^{2}}\right) & \text { if }|d x| \leq N \sigma \text { and } R_{h u b} \leq|\mathbf{r}| \leq R \\ 0 & \text { otherwise }\end{cases}
$$

with cells closer to the central plane of the turbine region having the largest weights and, therefore, contributions in the proceeding calculations. Using this method, any number of turbine regions can be represented simultaneously without a significant increase in computational effort. The sensitivity of the model to various parameters is discussed in Section 4. Note that, for simplicity, the presented model considers the weighting inside the turbine region to be uniform in the radial direction and for all cells outside of the turbine region the contribution to the local velocity is zero.

\subsection{Orientation}

For generality, the turbine model has been developed to allow the turbine to be placed in any orientation relative to the coordinate system of the computational domain. This is achieved via the orientation matrix, $\mathbf{Q}_{0}$, defined as

$$
\mathbf{Q}_{0}(\alpha, \beta, \gamma)=\mathbf{R}_{x}\left(\frac{\alpha}{2}\right) \mathbf{R}_{y}\left(\frac{\beta}{2}\right) \mathbf{R}_{z}(\gamma) \mathbf{R}_{y}\left(\frac{\beta}{2}\right) \mathbf{R}_{x}\left(\frac{\alpha}{2}\right)
$$




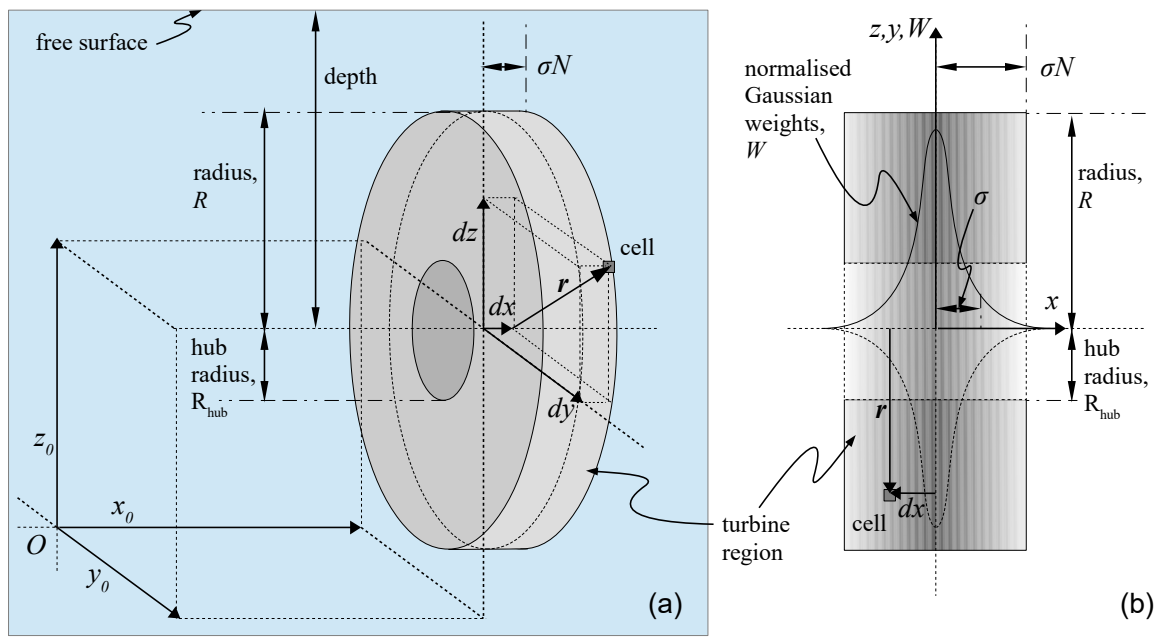

Figure 1: Schematic representation of a) the 'turbine region' and b) the Gaussian weighting function used in the turbine model.

where $\mathbf{R}_{x}, \mathbf{R}_{y}, \mathbf{R}_{z}$ are matrices defining a rotation about the global $x, y$ and $z$ axes respectively. These are defined as

$$
\mathbf{R}_{x}=\left(\begin{array}{ccc}
1 & 0 & 0 \\
0 & \cos (\alpha) & -\sin (\alpha) \\
0 & \sin (\alpha) & \cos (\alpha)
\end{array}\right)
$$

$$
\mathbf{R}_{y}=\left(\begin{array}{ccc}
\cos (\beta) & 0 & \sin (\beta) \\
0 & 1 & 0 \\
-\sin (\beta) & 0 & \cos (\beta)
\end{array}\right)
$$

$$
\mathbf{R}_{z}=\left(\begin{array}{ccc}
\cos (\gamma) & -\sin (\gamma) & 0 \\
\sin (\gamma) & \cos (\gamma) & 0 \\
0 & 0 & 1
\end{array}\right)
$$

where $\alpha, \beta$ and $\gamma$ are the angles of roll, pitch and yaw respectively.

A new coordinate system, based on the orientation matrix, is then generated and each cell in the domain is assigned a new set of coordinates in the rotated system according to

$$
\mathbf{d x}^{\prime}=\left(d x^{\prime}, d y^{\prime}, d z^{\prime}\right)=\mathbf{Q}_{0}^{T} \cdot \mathbf{d} \mathbf{x}
$$

The turbine weighting is then calculated (as described in Section 3.2) but with $\mathbf{d x}$ replaced by $\mathbf{d x} \mathbf{x}^{\prime}$.

\subsection{Free Stream Velocity Calculation}

As described in Section 3.1, the free stream velocity, $u_{\infty}$, is required to calculate the thrust on, and power generated by, the turbine. However, in 
general and particularly in wave and current cases, $u_{\infty}$ is time-varying and is not known in advance. Therefore, a method to determine the instantaneous free stream velocity from the known velocity field, local to the turbine region, has been developed. Using the weighting function, $W$, as described in Section 3.2, the weighted average relative velocity, $\mathbf{u}_{a v}$ in the turbine region can be calculated using

$$
\mathbf{u}_{a v}=\left(\frac{1}{V} \sum_{i=1}^{N} \mathbf{u}_{i} V_{i} W_{i}\right)-\mathbf{v}_{h u b},
$$

where $N$ is the total number of cells in the turbine region, $\mathbf{v}_{h u b}$ is the velocity of the turbine and $V$ is the total weighted volume of the turbine region based on the volume of each cell, $V_{i}$,

$$
V=\sum_{i=1}^{N} V_{i} W_{i} .
$$

The local speed in the axial direction, $u_{t}$, is then determined using

$$
u_{t}=\left|\mathbf{u}_{a v} \cdot \mathbf{x}_{a x i s}\right|,
$$

where

$$
\mathbf{x}_{\text {axis }}=\mathbf{Q}_{0}^{T} \cdot \hat{\mathbf{x}},
$$

is a unit vector parallel to the axis of the turbine and $\hat{\mathbf{x}}$ is a unit vector in the global x-direction. For a turbine with known axial induction factor, $a$, or thrust coefficient, $C_{t}$, the instantaneous free stream velocity is then calculated using a rearrangement of equation (1).

The instantaneous thrust on the turbine, $T$, and the instantaneous power generated, $P$, can then be calculated using equations (2) and (3) respectively.

\subsection{Update Momentum Equation}

Assuming laminar flow and neglecting surface tension, the incompressible (unsteady) RANS equations take the following form

$$
\frac{\partial(\mathbf{u})}{\partial t}+\nabla \cdot(\mathbf{u u})=-\nabla \frac{p}{\rho}+\nabla^{2}(\nu \mathbf{u})+\mathbf{g}+\mathbf{T},
$$

$$
\nabla \cdot \mathbf{u}=0,
$$

where $p$ is the pressure, $\rho$ is the fluid density, $\mathbf{u}=(u, v, w)$ is the fluid velocity, $\nu$ is the kinematic fluid viscosity, $\mathbf{g}$ is acceleration due to gravity and $\mathbf{T}$ is the momentum sink due to the presence of the turbine.

To achieve coupling between the calculated thrust on the turbine, $T$, and the associated momentum sink in the fluid, $\mathbf{T}$, the thrust force per unit mass is distributed across the turbine region using the weighting function, $W$, (from Section 3.2) such that

$$
\mathbf{T}_{i}= \pm \frac{T V_{i} W_{i}}{\rho V} \mathbf{x}_{\text {axis }},
$$


where \pm is positive for flow in the direction of the turbine axis or negative for reversed flow.

The governing equations for the fluid (equation 18) can then be solved for the next time step (transient) or iteration (steady-state) and the processes described in Sections 3.2-3.5 repeated until the maximum time (transient) or convergence (steady-state) is reached.

\section{Steady-State Analysis}

In this section, the sensitivity of the turbine model, to the various implementation parameters discussed above, is demonstrated using the single phase, steady-state solver simpleFoam. For this analysis, a single static turbine (i.e. $\left.\left|\mathbf{v}_{h u b}\right|=0\right)$ is considered, in isolation. The objective here is to demonstrate the robustness of the model as well as verify that the model returns the expected results for idealised cases.

The modelling technique described in Section 3, relies fundamentally on predicting the free stream velocity, $u_{\infty}$, from the local velocity in the turbine region, $u_{t}$. Hence, to quantify the accuracy of the model and assess the sensitivity of the approach to the key parameters, comparisons are made between the predicted value of $u_{\infty}$ and the user defined value at the inlet. The convergence criteria are kept constant throughout this analysis and are set to have maximum residual values of $10^{-4}$ and $10^{-5}$ for the pressure and velocity fields respectively.

\subsection{Initial Setup}

Unless stated otherwise, the sensitivity test cases use a turbine model with a radius of $2 \mathrm{~m}$ (the hub radius is set to zero), a $C_{t}=0.9, N=2$, and $\sigma=0.15$. The prescribed free stream velocity is $1 \mathrm{~m} \mathrm{~s}^{-1}$.

The required cell size around the turbine was evaluated using a mesh convergence study. The initial numerical domain was a $200 \times 20 \times 20 \mathrm{~m}$ cuboid consisting of cubic cells (side length $=0.5 \mathrm{~m}$ ) and a $5 \mathrm{~m}$ cubic region around the turbine, refined by one level using the octree refinement strategy [9]. The refinement in the turbine region was then incrementally increased until the value of the predicted free stream velocity changed by less than $0.1 \%$ between meshes. This was found to occur for cells $3.125 \mathrm{~cm}$ in length (4 levels of refinement). All remaining simulations, in the sensitivity analysis, use cells of this size in the turbine region unless otherwise stated.

To optimise the dimensions of the computational domain, with respect to a reduction in both the blockage effects arising from the boundaries and the required computational effort, a series of tests focusing on the inlet, outlet and side wall distances were performed. By incrementally increasing one of these distances (whilst fixing the other two), the minimum distance from the turbine was determined based on convergence of the predicted free stream velocity. By applying this method to all boundaries, a $100 \times 100 \times 100 \mathrm{~m}$ numerical domain was selected for the sensitivity analysis, running from $-50 \mathrm{~m}$ to $50 \mathrm{~m}$ in each dimension, with the centre of the turbine located at the origin (Figure 2). 

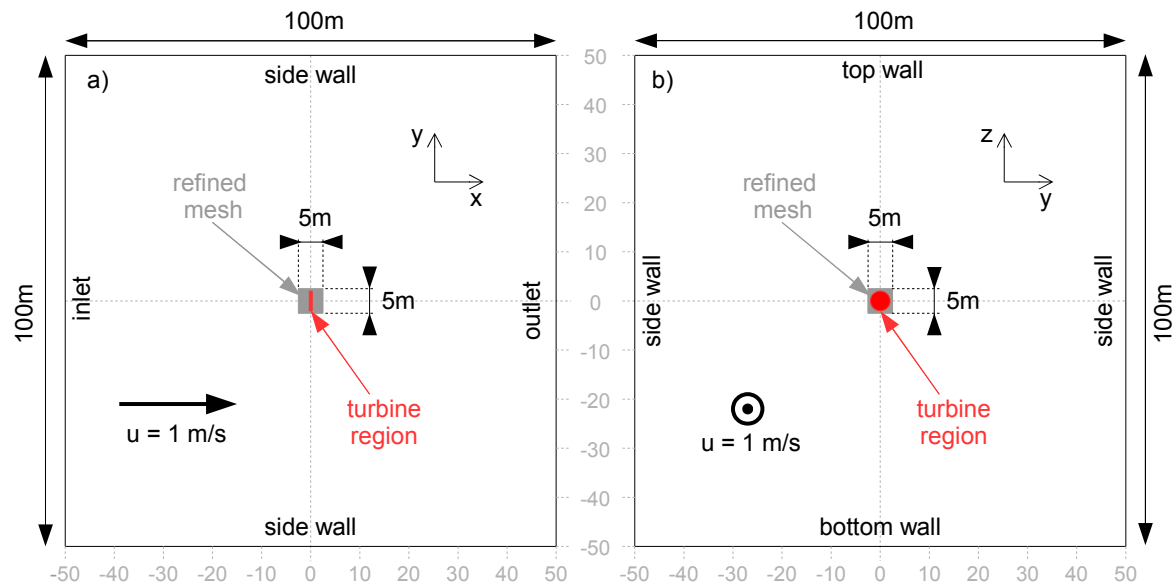

Figure 2: Sketch of the numerical domain used for the steady-state sensitivity analysis in a) the $x-y$ plane at $z=0$, and b) the $y-z$ plane at $x=0$, with the turbine region indicated in red.

The side walls of the domain are considered to be solid and have no-slip boundary conditions applied to them, the inlet boundary has the free stream velocity prescribed as a boundary condition and the outlet has zero gradient conditions to represent flow leaving the domain. The initial condition is the prescribed free stream velocity used at the inlet, and turbulence modelling has not been considered in this analysis.

\subsection{Width Parameters}

Actuator theory is based on an infinitesimally thin disc $[11,18]$ and therefore, in the present model, a delta function to describe the turbine width would likely give the most accurate solution. This would be possible in finite volume methods, if the turbine was fixed and aligned perfectly with the cell centres (or faces). However, in the present study, the turbine model is coupled to a floating structure which (as well as having arbitrary mesh motion and structure), in general, has arbitrary alignment with the mesh. It is, therefore, necessary to assign a finite region in which to ascertain the local flow velocity and apply the corresponding momentum sink, as an infinitesimally thin region would not perform well in cases (or time steps) in which there is a misalignment with the computational mesh. In this section, the sensitivity of the model to the width of this finite turbine region, $2 N \sigma$, is considered.

A series of simulations were performed using different combinations of $N$ and $\sigma$ and, using the converged solution for $u_{\infty}$, the error [\%] was calculated with respect to the prescribed inlet flow speed $\left(1 \mathrm{~m} \mathrm{~s}^{-1}\right)$.

Figure 3a shows the error, as a function of $\sigma \sqrt{N}$, for the case in which the central plane of the turbine is coincident with cell faces. For higher values 

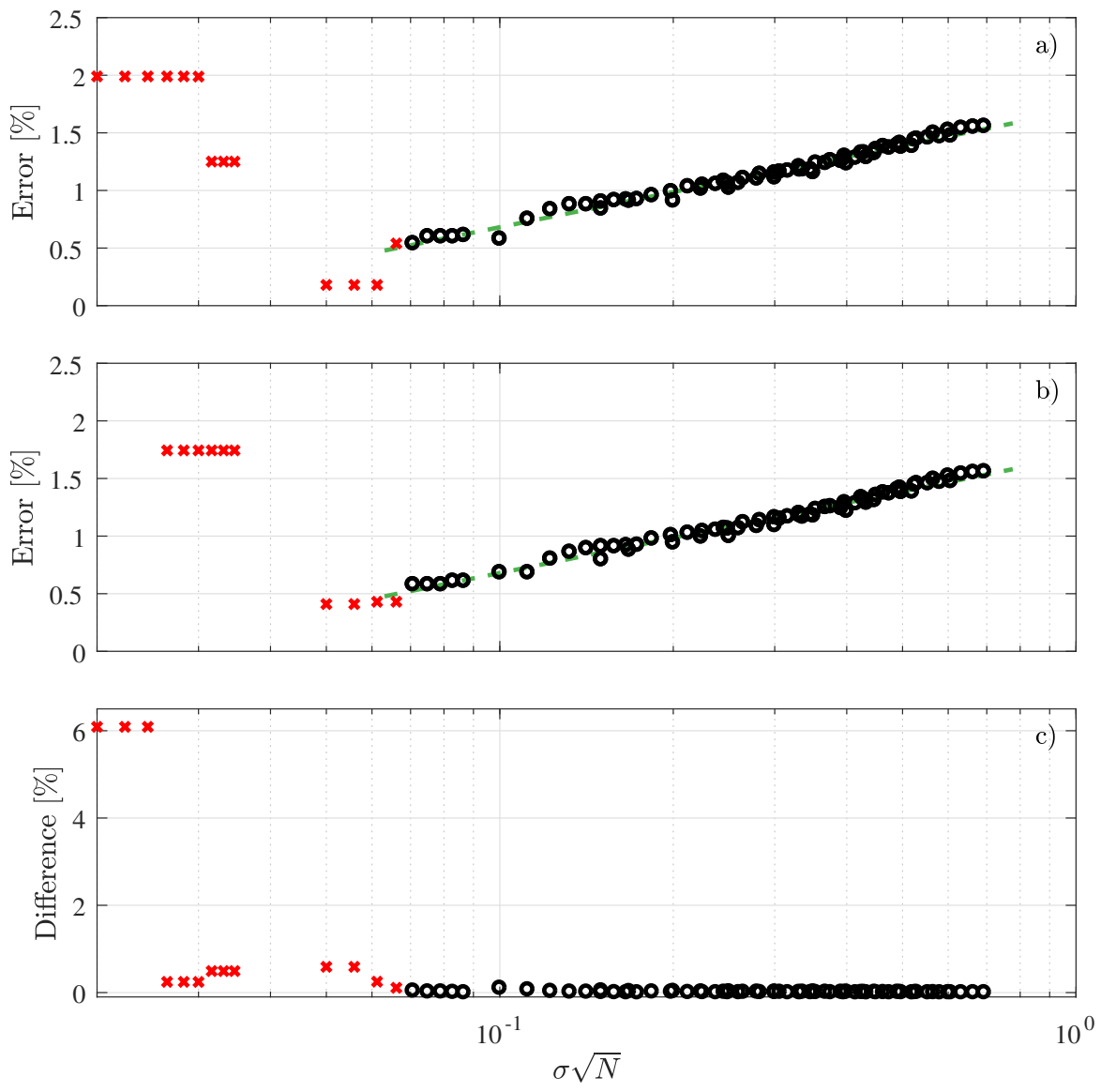

Figure 3: Error [\%] in $u_{\infty}$ as a function of $\sigma \sqrt{N}$, when the turbine is aligned with a) cell faces, b) cell centres. Values which follow a log law $(--)$ are indicated as circles (o) otherwise they are represented by crosses $(\times)$. Also shown (c) is the difference [\%] between the solutions obtained with the turbine aligned with cell faces and with cell centres.

of $\sigma \sqrt{N}$ (marked with o), as the width moves towards the ideal case of an infinitesimally thin disc, the error decreases with a logarithmic trend (indicated by the dashed line $(--)$ ). However, below a certain 'cut-off' the logarithmic trend breaks down $(\times)$; the error initially decreases, before rapidly increasing. The rapid increase in error at low $\sigma \sqrt{N}$ values is a consequence of the total width of the turbine region approaching the width of a single cell. Based on this, it seems that the optimal turbine width would be the value at which this cut-off occurs, i.e. small enough to minimise the error, but large enough that the results lie in the well-behaved, logarithmic region. For the combination of mesh, turbine and flow speed used here, the cut-off value was found to be $\sigma \sqrt{N}=0.07$, with an error of $1 \%$ or less for $0.07 \leq \sigma \sqrt{N} \leq 0.11$.

In general, the turbine's central plane will be positioned arbitrarily relative 
to each mesh cell and hence the solution must be independent of this parameter. Figure $3 \mathrm{~b}$ shows the error for the case in which the central plane of the turbine is coincident with the mesh cell centres. With respect to the staggering of the mesh cells relative to the turbine central plane, this represents the most extreme alternative to the case used above which was coincident with the mesh faces. From Figure 3b, it can be seen that the error for a turbine central plane coincident with the cell centres has the same trend as that for that coincident with the cell faces (Figure 3a).

The difference between the two solutions is presented in Figure 3c. For low values of $\sigma \sqrt{N}$ there are unacceptable differences between the solutions; it is anticipated that, in the general case of a turbine moving arbitrarily through the mesh, these would generate unphysical fluctuations in critical values (e.g. the thrust on the turbine). Although small differences can be observed around $\sigma \sqrt{N}=0.1$, the differences between the two solutions are much smaller in the logarithmic region. This is due to the increased turbine width distributing the weighting over more cells and reducing the sensitivity to single values (including the difference between coincidence with a cell centre or a cell face).

The results presented in Figure 3 imply that the model is not overly sensitive to the coincidence of the mesh cells and the turbine central plane, provided $\sigma \sqrt{N}$ is reasonably large. This is essential for the model to be successful in a moving mesh simulation. However, the results also demonstrate that for high levels of accuracy the turbine region, i.e. $\sigma \sqrt{N}$, should be kept relatively small. As a compromise, in this particular case, $N$ and $\sigma$ have been chosen to achieve $\sigma \sqrt{N}=0.11$ giving an error of around $1 \%$, whilst maintaining a solution that is suitably independent of the coincidence of the mesh cells and turbine central plane.

\subsection{Mesh Dependency}

As mentioned above, the error in the predicted value of $u_{\infty}$ appears to have some mesh dependence at low $\sigma \sqrt{N}$ values. Consequently, further simulations were performed, with varying $\sigma$ and $N$, with the mesh in the region of the turbine one octree level finer $(1.5625 \mathrm{~cm})$ or one level coarser $(6.25 \mathrm{~cm})$ compared to the mesh used in Section 4.2 (see Table 1 for details).

Figure 4 shows the error [\%], as a function of $\sigma \sqrt{N}$, for each of the three mesh resolutions (original $(\circ)$, finer $(\Delta)$ and coarser $(\diamond)$ ). The dashed lines represent the logarithmic trends of the mesh in the corresponding colour. The

Table 1: Mesh resolution, aspect ratio, octree level and total size used in each of the steadystate, static cases.

\begin{tabular}{lccccc}
\hline Mesh & \multicolumn{2}{c}{ Background } & \multicolumn{2}{c}{ Refined Region } & Total Cells \\
& $\Delta x[\mathrm{~m}]$ & AR & Oct. lvl. & $\Delta x[\mathrm{~m}]$ & \\
\hline \hline Coarse & 0.5 & 1 & 3 & 0.0625 & $8.5 \mathrm{M}$ \\
Medium & 0.5 & 1 & 4 & 0.0313 & $12.2 \mathrm{M}$ \\
Fine & 0.5 & 1 & 5 & 0.0156 & $40.7 \mathrm{M}$ \\
\hline
\end{tabular}




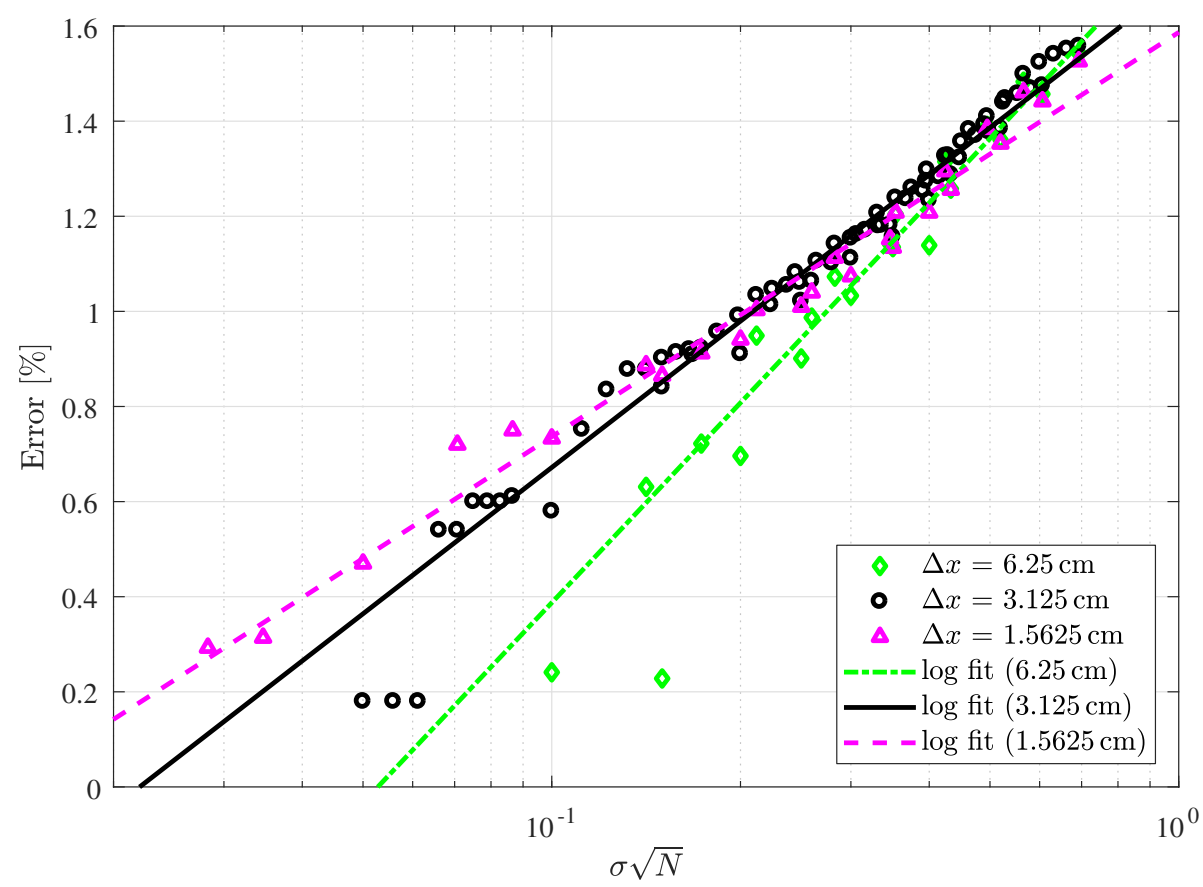

Figure 4: Comparison of the error [\%] as a function of $\sigma \sqrt{N}$ for three different mesh resolutions: $\Delta x=6.25 \mathrm{~cm}(\diamond), 3.125 \mathrm{~cm}(\circ)$ and $1.5625 \mathrm{~cm}(\Delta)$. The lines represent the logarithmic error trend for each case dash-dotted, solid, and dashed, respectively.

gradient of the trend lines decreases with increasing resolution, as does the cutoff width, $\mathcal{C}$, defining the end of the logarithmic region, i.e. for finer meshes, the logarithmic region holds for much lower $\sigma \sqrt{N}$ and so higher accuracies can be achieved before the model becomes too sensitive to motion through the mesh. For higher $\sigma \sqrt{N}$ the solutions from the three meshes are very similar, indicating mesh independence. It is unclear whether the logarithmic regions continue for much higher values of $\sigma \sqrt{N}$, but it is unlikely that a width greater than those considered here would be beneficial due to the increased error.

Based on the logarithmic trends observed in Figure 4, the error in the predicted value of $u_{\infty}$ takes the form

$$
\mathcal{E}(\%)=\mathcal{A} \ln (\sigma \sqrt{N})+\mathcal{B}, \quad \text { if } \quad \sigma \sqrt{N} \geq \mathcal{C}
$$

where $\mathcal{A}, \mathcal{B}$ and $\mathcal{C}$ are all functions of mesh resolution $\Delta x$.

Figure 5 shows that $\mathcal{A}$ and $\mathcal{C}$ (and to a reasonable degree $\mathcal{B}$ ) are linear functions of mesh resolution $\Delta x$, and for this case

$$
\mathcal{A}=5.0807 \Delta x+0.288, \quad \mathcal{B}=4.0155 \Delta x+1.5418, \quad \mathcal{C}=2.56 \Delta x-0.01 .
$$

The coefficients in equation (22) are likely to be functions of the turbine diameter $(4 \mathrm{~m})$ and the incident flow speed $\left(1 \mathrm{~m} \mathrm{~s}^{-1}\right)$, however, by combining equations 

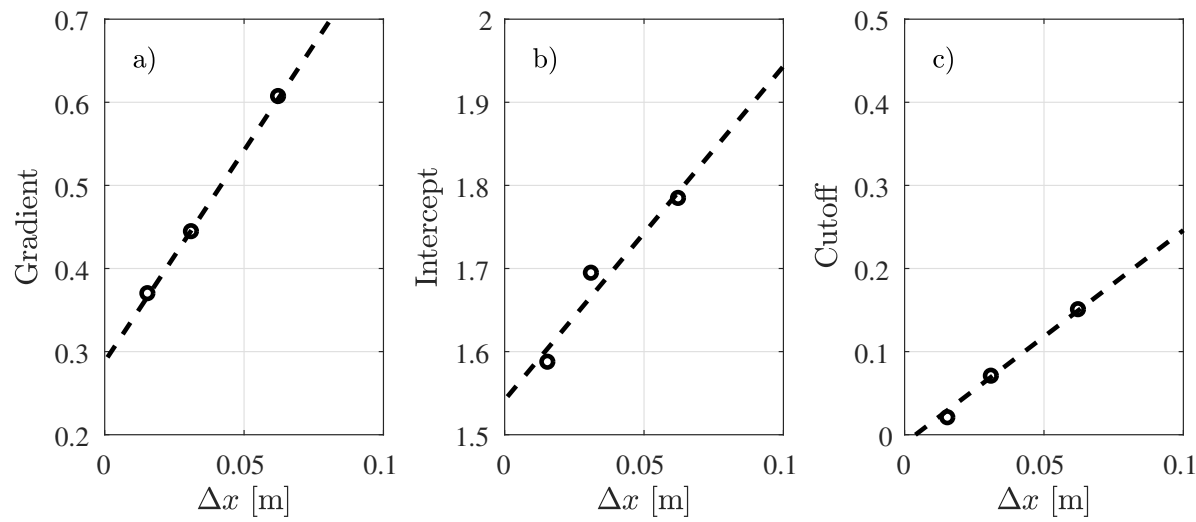

Figure 5: Properties of the logarithmic error trend as a function of mesh resolution: a) gradient, b) intercept, and c) the minimum $\sigma \sqrt{N}$ cut-off value for which the trend holds.

(21) and (22) the error in this case could be estimated for any given mesh discretisation, allowing a suitable value of $\sigma \sqrt{N}$ to be chosen.

\subsection{Flow Speed}

Over a tidal cycle, a turbine will experience a wide range of flow speeds; furthermore, for floating tidal energy applications the turbine will be subject to a combination of both tidal currents and waves, i.e. oscillatory flow. Consequently, in order to model a full floating tidal energy concept, in realistic conditions, it is vital that the performance of the turbine model is not overly sensitive to the flow speed.

To assess the performance of the present model as a function of free stream velocity, $u_{\infty}$, a series of simulations were run with different prescribed flow speeds (in the range $0.25-4 \mathrm{~m} \mathrm{~s}^{-1}$ ). Figure 6 a presents the predicted $u_{\infty}$ values against the prescribed inlet velocities, with the red, dotted line representing perfect prediction. The predicted and prescribed values generally agree very well, although as the flow speed increases the deviation does appear to increase. Considering Figure 6b (which shows the error as a function of prescribed inlet speed), it is clear, however, that the relative error remains very similar throughout, i.e. $\approx 0.95 \%$ for all of the 16 flow speeds tested. It can therefore be concluded that, the model developed here performs equally well over the required range of incident flow speeds.

\subsection{Turbine Characteristics}

So far in this section, the turbine characteristics have been fixed to represent a generic turbine with a radius of $2 \mathrm{~m}$ and a thrust coefficient of 0.9 . In realistic applications, these parameters will be determined by the turbine manufacturer and, although it may be constant during operational conditions, the thrust coefficient could potentially change in order to produce favourable output characteristics or reduce the chance of damage to the generator at high flow 

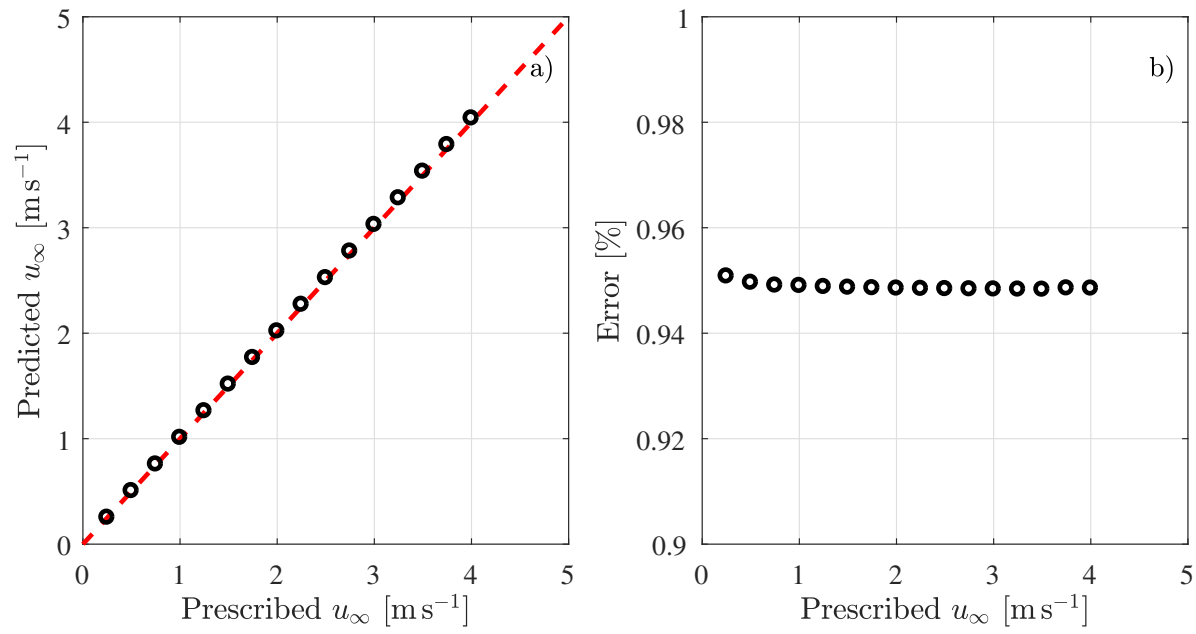

Figure 6: Sensitivity of the model to flow speed: a) predicted free stream value as a function of the prescribed value at the inlet, and b) the relative error as a function of prescribed flow speed.

speeds. Hence, for completeness, and generality, the performance of the model is assessed for turbines of different thrust coefficients, utilising the same mesh and simulation setup as presented earlier.

Figure 7 shows the error [\%] as a function of thrust coefficient, $C_{t}$. In this case: For low thrust coefficients $\left(C_{t}<0.65\right)$ the free stream velocity is slightly under-predicted; for high thrust coefficients $C_{t} \geq 0.65$ the free stream velocity is over-predicted. Further work is required to understand this behaviour for different turbine characteristics, flow speeds and domain sizes but it appears that, for all except the very highest $C_{t}$ values, the predicted free-stream velocity is well within $1 \%$ of the true value.

\section{Prescribed Motion Cases}

The aim of this work is to develop a turbine methodology that can be used in the simulation of entire floating tidal stream systems. In Section 4, the methodology is shown to predict with good accuracy the free stream velocity, and hence the thrust, in the case of a static turbine. However, when simulating the complete coupled system, the movement of the device (in any of six degrees of freedom) leads to a time-varying turbine position with arbitrary location and alignment with the numerical grid. This prevents the use of highly contrived meshes designed solely to capture the turbine well $[7,12,24]$ and requires a methodology capable of seamlessly transitioning through the mesh without causing numerical instabilities. In this section the ability of the present method, to meet this requirement, is demonstrated via a series of test cases in which the turbine is given prescribed motion through the computational domain. 


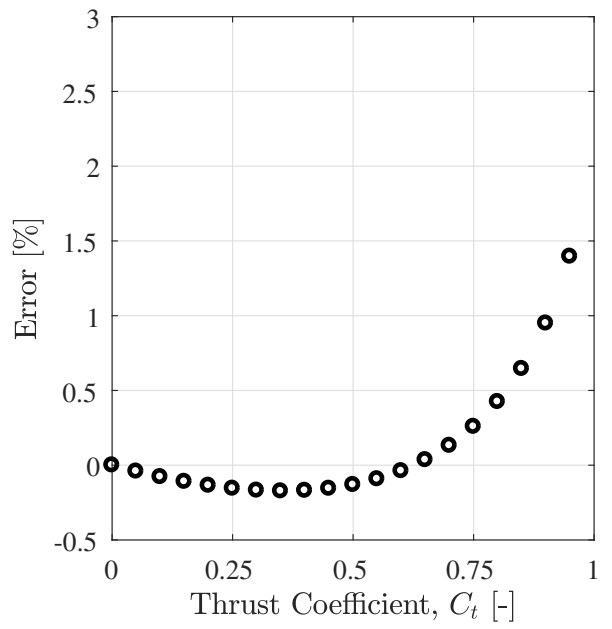

Figure 7: Sensitivity of the model accuracy to turbine thrust coefficient, $C_{t}$.

In each case, a turbine with $R=2 \mathrm{~m}, C_{t}=0.9, N=2$ and $\sigma=0.15$ is moved with prescribed velocity, $\mathbf{v}$, through initially still water and the predicted free stream velocity is again compared to the true $u_{\infty}$. The tests were run for $60 \mathrm{~s}$ using the modified transient solver pisoFoam (see Section 3) and the same $100 \times 100 \times 100 \mathrm{~m}$ domain as that described in Section 4 (see Figure 2). Only the refined region in the path of the turbine, its initial location/orientation and prescribed velocity vary in each case. The boundary conditions are the same as described in Section 4 (with inflow speed of $0 \mathrm{~ms}^{-1}$ ), the initial conditions are zero flow conditions, and turbulence modelling has not been considered in this section.

In all cases, the speed of the turbine is ramped up to avoid effects arising from instantaneous movement of the turbine. The ramp up is described by the sinusoidal function

$$
\mathbf{v}_{\text {hub }}= \begin{cases}\frac{1}{2} \mathbf{v}\left[1-\cos \left(\frac{\pi}{t_{\text {ramp }}} t\right)\right] & \text { if } t<t_{\text {ramp }} \\ \mathbf{v} & \text { if } t \geq t_{\text {ramp }}\end{cases}
$$

where $t_{\text {ramp }}$ is the ramp up time (set to $20 \mathrm{~s}$ in this work) and the position of the turbine is updated based on the integral of this function.

\subsection{Constant Linear Velocity}

In the first two test cases, the turbine is given a constant velocity through the mesh, $\left|\mathbf{v}_{\text {hub }}\right|=1 \mathrm{~ms}^{-1}$. These cases are considered to be equivalent to the idea of a physical towing tank and it is anticipated that the turbine behaves the same as if it were fixed in uniform flow with velocity equal to the prescribed motion, i.e. the relative flow over the turbine is the same. 

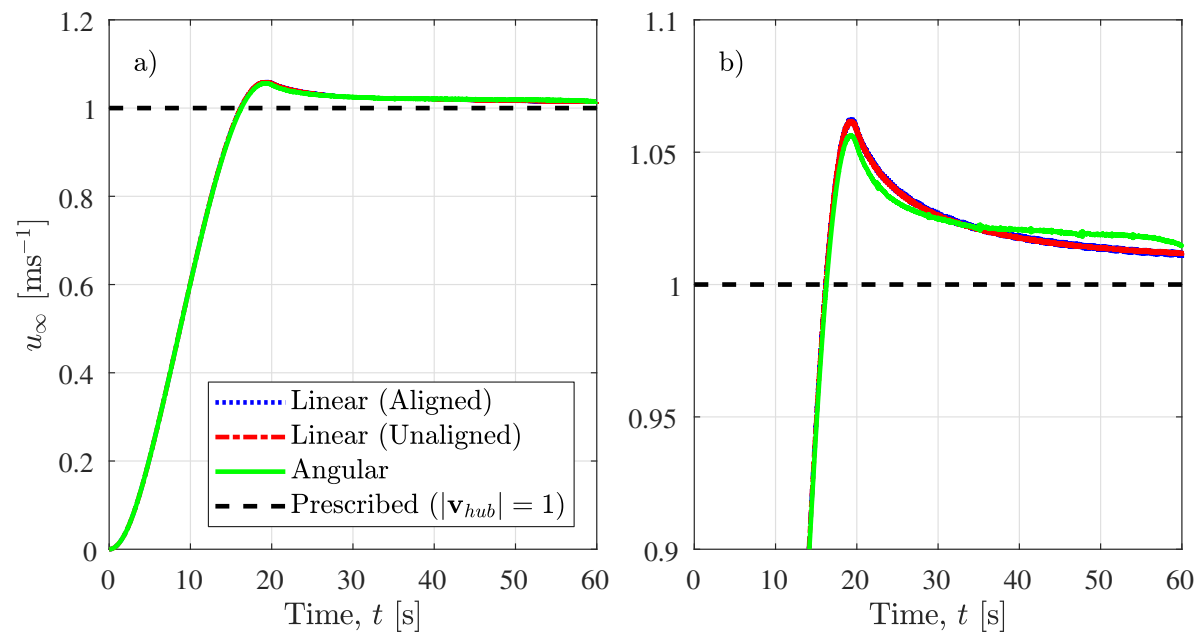

Figure 8: Time series of the prediction of $u_{\infty}$ in the aligned $(\cdots)$, misaligned $(-\cdot-)$ and angular $(-)$ prescribed velocity cases, along with the prescribed velocity $(--)$. Both the full time series (a) and a magnified view (b) are presented.

Table 2: Initial turbine position, orientation and refined region for each of the prescribed motion cases. The mesh resolution is given by the 'coarse' mesh in Table 1, and the refined region is $[-2.5,2.5]$ in the $z$ direction in all cases.

\begin{tabular}{lcccccccc}
\hline Case & \multicolumn{2}{c}{ Init. Hub Pos. } & \multicolumn{3}{c}{ Refined Region } & \# Cells & Timestep \\
& $x$ & $y$ & $\gamma$ & $x$ & $y$ & $\gamma$ & & $\Delta t[\mathrm{~s}]$ \\
\hline \hline Aligned & 30 & 0 & $0^{\circ}$ & {$[-31,31]$} & {$[-2.5,2.5]$} & $0^{\circ}$ & $14.6 \mathrm{M}$ & 0.01 \\
Misaligned & 18 & -18 & $45^{\circ}$ & {$[-22,40]$} & {$[-20,20]$} & $45^{\circ}$ & $14.6 \mathrm{M}$ & 0.01 \\
Angular & 0 & -8 & $0^{\circ}$ & {$[-13,13]$} & {$[-13,13]$} & $0^{\circ}$ & $19.1 \mathrm{M}$ & 0.01 \\
\hline
\end{tabular}

The first test demonstrates the case of the turbine moving parallel to the $x$-axis, i.e. aligned with the mesh. At time $t=0 \mathrm{~s}$ the centre of the turbine is located at $\mathbf{x}_{h u b}=(30,0,0) \mathrm{m}$ and, after the period of ramp up, the turbine moves with the constant prescribed velocity, $\mathbf{v}_{h u b}=(-1,0,0) \mathrm{ms}^{-1}$. The mesh in the region along the path of the turbine (Table 2) is refined by three octree levels $(\Delta x=0.0625 \mathrm{~m}$ ), which (based on the information in Section 4) gives an anticipated error in the predicted free stream velocity of approximately $1 \%$.

Figure $8 \mathrm{a}$ presents a time series of the predicted free stream velocity, $u_{\infty}$, for the aligned case $(\cdots)$ and the final, prescribed speed, i.e. the true solution $(--)$. The initial ramp up of the turbine velocity can be observed, along with an over-shoot $(\sim 6 \%)$ as the ramp up period ends. After this, the prediction converges towards the anticipated solution with an error of approximately $1 \%$ (observed at time $t=60 \mathrm{~s}$ ). Crucially, the prediction of $u_{\infty}$ is relatively smooth, indicating that the present methodology works well for turbines moving parallel to the axes of the mesh. There are some very small fluctuations $\left(\sim 0.001 \mathrm{~ms}^{-1}\right)$ in the prediction that can be observed when considered more closely (Figure 8b), which are likely due to the instantaneous position of the turbine (see Section 4.2) 
but these are considered to be negligible and are not expected adversely to affect the stability of the simulation.

The second case considers turbine motion that is not aligned with the axes of the mesh. This is achieved by rotating the turbine axis by $45^{\circ}$ and prescribing a constant velocity $\mathbf{v}_{h u b}=(-0.707,0.707,0) \mathrm{ms}^{-1}$. The turbine is initially located at $\mathbf{x}=(18,-18,0)$ and the mesh along the path of the turbine (Table 2$)$ is again refined by three octree levels (again with an anticipated error of 1\%). The predicted value of $u_{\infty}$, in this case, is presented in Figure 8a $(-\cdot-)$. The time series is very similar to that observed for the aligned case; after the initial ramp up period, the prediction overshoots before converging to $1 \%$ of the prescribed speed by $t=60 \mathrm{~s}$. The error is marginally larger than that observed in the aligned case but, interestingly, the fluctuations in the prediction are smaller (Figure $8 \mathrm{~b}$ ). This is thought to be caused by the 'mis-alignment' of the turbine: In the aligned case, the edge of the turbine region crosses cell faces at all points simultaneously; hence, any slight differences between the cells also contribute to the solution simultaneously resulting in a more noticeable change. In the nonaligned case, the edge of the turbine region crosses the cell faces arbitrarily and hence the differences contribute asynchronously resulting in lower fluctuations.

\subsection{Constant Angular Velocity}

In Section 5.1 the present turbine methodology is shown to be robust and accurate, when moving at a constant linear velocity (either aligned and misaligned with the computational mesh). Floating tidal stream devices, however, are capable of moving in all six degrees of freedom and so, it is crucial that the methodology can also accommodate rotational motion through the mesh.

The third prescribed motion test case considers the turbine rotating about the $z$-axis with a constant angular velocity, $\omega=0.125 \mathrm{rad} \mathrm{s}^{-1}$. The turbine's velocity is given by the instantaneous tangential velocity (at the turbine hub), $\mathbf{v}_{\text {hub }}=\omega \mathcal{R}$, where $\mathcal{R}$ is the orbital radius. At each time step the value of $\gamma$ has been updated (relative to the centre of the orbit), and $\mathbf{R}_{z}$ (equation 12) is applied an additional time in equations (13) and (17) to capture the rotation of the turbine. In this case $\mathcal{R}=8 \mathrm{~m}$ and so $\left|\mathbf{v}_{h u b}\right|=1 \mathrm{~ms}^{-1}$. One complete orbit takes approximately $60 \mathrm{~s}$, ensuring the turbine region does not interact with the wake from the previous orbit. The mesh along the path of the turbine (Table 2) is refined to the same discretisation as in the linear velocity cases.

The predicted free stream velocity in the rotating case is presented in Figure $8 \mathrm{a}(-)$. The results show the same trend as in the linear velocity cases: An initial over-shoot in the prediction after the ramp up period, before converging to within $2 \%$ of the expected solution. This error is slightly larger than in the linear velocity cases, however, this might be anticipated as the underlying theory behind the expected solution is based on uniform flow across the turbine (and this is not true in this case). Crucially, again, there are only negligible fluctuations in the predicted solution (Figure 8b) indicating that the present methodology performs well even with arbitrary mesh alignment and rotational motion through the computational mesh. 


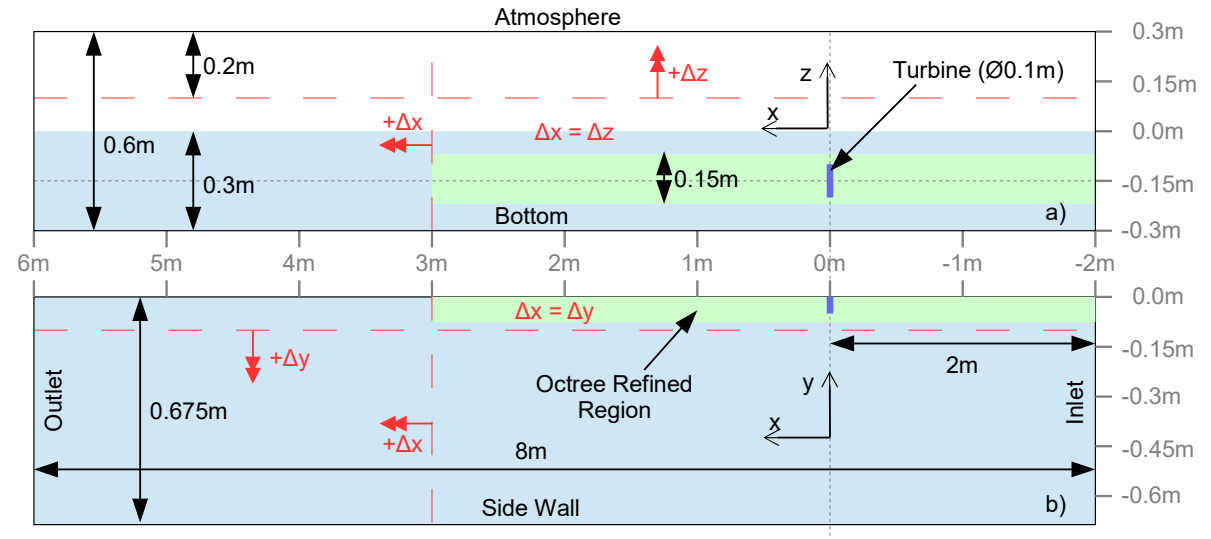

Figure 9: Numerical domain used for the two-phase simulations, in the $x-z$ (a), and $x-y$ (b) planes. Information regarding the mesh resolution is indicated in red, with double headed arrows representing mesh grading. The green shading indicates the refined region.

\section{Velocity Deficit Validation}

In Sections 4 and 5 the numerical model is shown to be robust and capable of capturing turbine loads when moving through a mesh, which is the primary motivation for the model. However, a secondary objective is to determine whether the turbine models influence on the fluid is captured accurately. Therefore, in this section the model is validated against existing experimental data for the velocity deficit behind a porous disc [31, 14]. These experiments were conducted in the Chilworth research laboratory flume at the University of Southampton, which is $21 \mathrm{~m}$ in length, $1.35 \mathrm{~m}$ wide and used a nominal water depth of $0.3 \mathrm{~m}$ [31]. Small scale discs $(\varnothing 0.1 \mathrm{~m}, 0.001 \mathrm{~m}$ width $)$ of varying porosity $(C t=0.61$, 0.86 and 0.94 ) were evaluated, with wake profile measurements taken at a point location (varied between runs) using an Acoustic Doppler Velocimeter (ADV).

The interFoam solver (see Section 3) coupled with the developed turbine model is used to simulate the problem. Current speeds of $0.2487 \mathrm{~ms}^{-1}$ are generated using the expression based boundary condition and relaxation zone technique provided as part of the waves2Foam toolbox [17]. The $k-\omega$ SST turbulence closure scheme [28] is used to model the turbulent effects. For computational efficiency, the numerical model simulates one half of the flume (and disc), assuming that the flow is symmetric at the $y=0$ plane. The water depth is set to $0.3 \mathrm{~m}(-0.3 \leq z \leq 0.3)$ and the tank width is $0.675 \mathrm{~m}(-0.675 \leq y \leq 0)$, consistent with the experiments [31]. The simulated length of the tank is set to $8 \mathrm{~m}(-2 \leq x \leq 6)$, to accommodate an inlet region $(-2 \leq x \leq 0)$, a working region $30 \mathrm{D}$ in length $(0 \leq x \leq 3)$ and a relaxation zone $(3 \leq x \leq 6)$. The initial mesh is designed such that the Aspect Ratio (AR) is set to 1 in the working region, with a mesh resolution of $\Delta x=0.01 \mathrm{~m}$ (Figure 9). Mesh grading is used to reduce computational cost in all directions: $x \geq 3, y \leq-0.1$ and $z \geq 0.1$. 

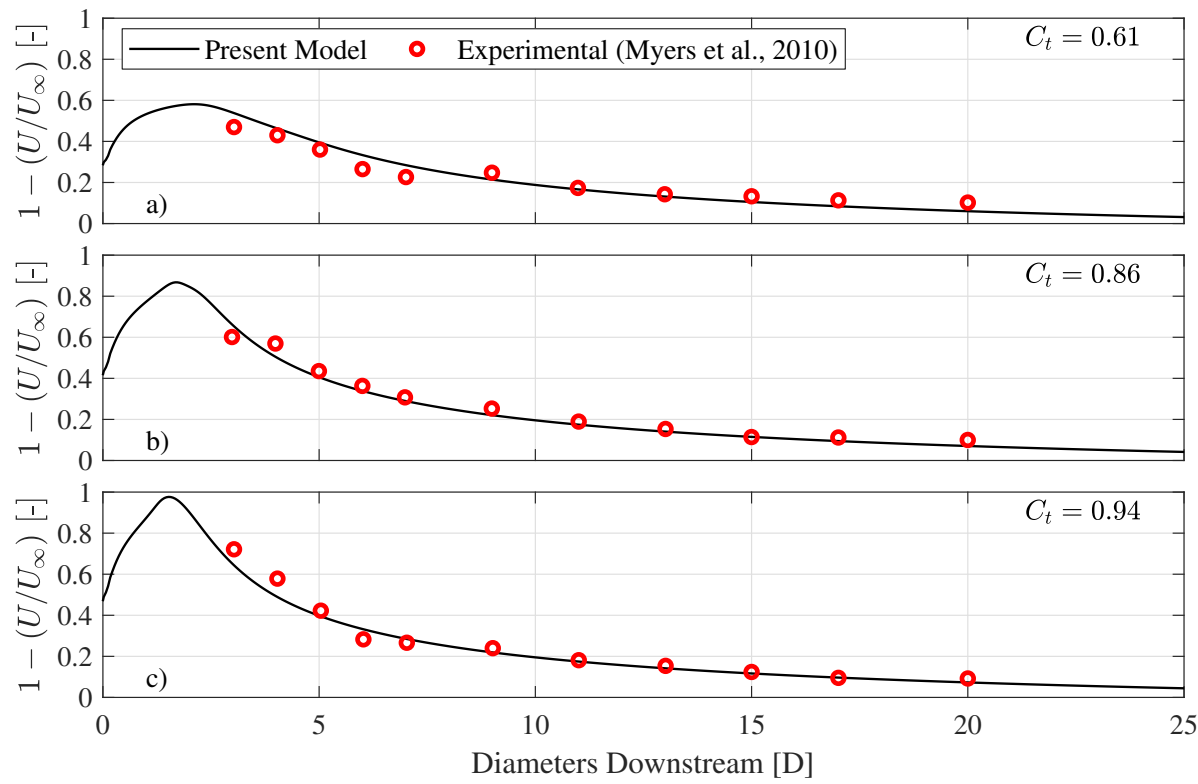

Figure 10: Comparison of experimental [31] (०) and numerical predictions ( - ) of centreline, horizontal velocity deficit profiles for $C_{t}=0.61$ (a), 0.86 (b) and 0.94 (c).

Two levels of additional octree refinement [10] are used in the region of the wake of the turbine $(-2 \leq x \leq 3$, radius $0.1 \mathrm{~m}, \Delta x=0.0025 \mathrm{~m})$.

The disc is centred at $x=0, z=-0.15$, with $R=0.05 \mathrm{~m}, \sigma=0.005$ and $N=2$, which gives an expected error of approximately $1 \%$ based on the results in Section 4, and each case is run for $120 \mathrm{~s}$ of simulation time. The inlet and outlet boundaries for velocity are both set to the prescribed free stream velocity $\left(0.2487 \mathrm{~ms}^{-1}\right)$ in the water phase $\left(0 \mathrm{~ms}^{-1}\right.$ in the air phase). The top boundary is modelled as an atmosphere condition with a total pressure condition applied. The bottom and side boundaries are considered to be walls and hence are modelled with no-slip conditions. Wall functions are used for the turbulent parameters at these boundaries and hence mesh refinement is applied adjacent to these boundaries to achieve a suitable $y^{+}$value $\left(y^{+} \approx 40\right)$. The inlet turbulent conditions are determined based on an inlet turbulent intensity of $5 \%$, with zero gradient conditions applied at the outlet and atmosphere boundaries. The initial conditions for velocity and turbulence parameters is set to the values specified at the inlet.

Figure 10 presents a comparison of experimental (०) and numerical prediction $(-)$ for the disc's centreline $(y=0 \mathrm{~m}, z=-0.15 \mathrm{~m})$ velocity deficit profile as a function of diameters downstream, for $C_{t}=0.61$ (a), 0.86 (b) and 0.94 (c). In all cases, the numerical predictions agree well with the experimental data. The near wake region $(x \leq 5 D)$ was observed to increase with thrust coefficient in the experimental data. The numerical model captures this effect due to thrust coefficient well, with progressively increasing velocity deficit: the 

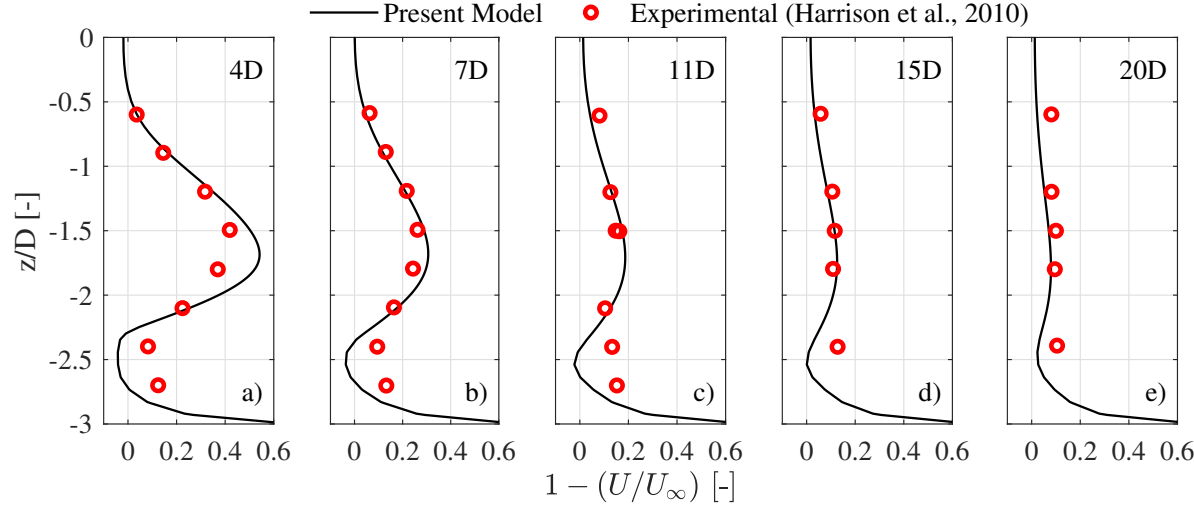

Figure 11: Comparison of experimental [12] (०) and numerical predictions ( - ) of vertical velocity deficit profiles for a $C_{t}=0.86$ disc, at $x=4 D$ (a), $7 D$ (b), $11 D$ (c), $15 D$ (d) and $20 D(\mathrm{e})$.

predictions of maximum velocity deficits are $0.58,0.87$ and 0.98 for $C_{t}=0.6$, 0.86 and 0.94 , respectively, although it should be noted that these can not be validated since the experimental campaign only considered positions for $x \geq 3 D$.

A comparison of the experimental (o) and numerical predictions ( - ) of vertical profiles is presented in Figure 11 for the $C_{t}=0.86$ at a number of horizontal locations: $x=4 D$ (a), $7 D$ (b), $11 D$ (c), $15 D$ (d) and $20 D$ (e). At $4 D$ in the experimental data, there is a region of high velocity deficit, which extends from $z / D \approx-2$ to $z / D \approx-1$, i.e. the position of the disc. This is also observed in the numerical predictions, and the maximum occurs slightly below the centreline of the disc at this location, which has also been observed in previous CFD studies of the wake structure behind an actuator disc in a marine environment $[4,34]$. Moving further away from the disc, the experimental data shows that this region reduces in magnitude and increases in height, which is also captured by the numerical model. However, the maximum value gets lower with increasing $x$ in the numerical predictions, which although not obvious in the point measurements presented in Figure 11, could be observed in spatial plots presented by Myers and Bahaj [31]. In this work, the behaviour is more clearly observed in spatial plots of the numerical data (Figure 12), and is consistent for each of the discs considered. The spatial plots also show that the wake distribution for $x \geq 8 D=0.8 \mathrm{~m}$ is very similar for the three discs. This indicates that the far wake structure is independent of the properties of the disc, and is in-line with the observations of Myers and Bahaj [31].

Overall, the numerical model captures velocity deficit to a similar standard as other numerical models $[12,4,34]$, and distributions are comparable with experimental data [31]. Therefore, it is concluded that the model would be suitable for investigating both the effect of the turbine on a structures motion, and the implications for the fluid flow, in future work. 

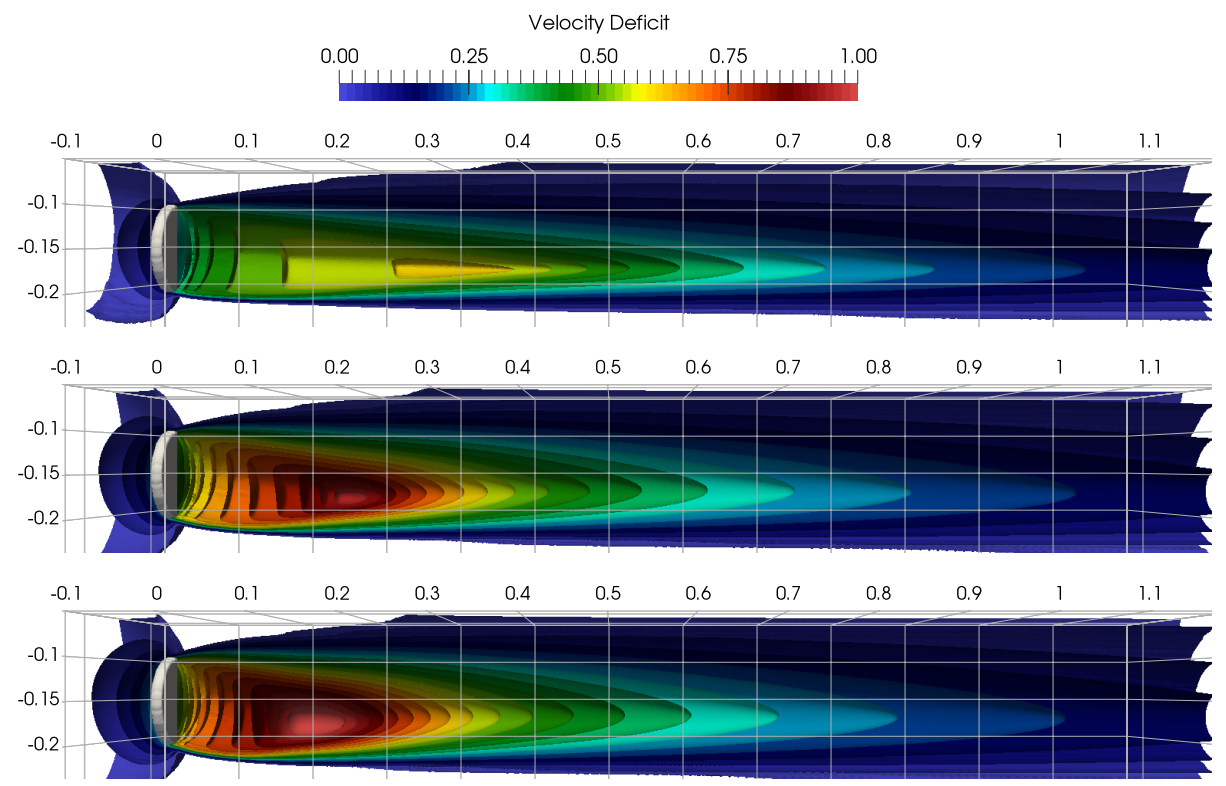

Figure 12: Velocity deficit contours for different turbine thrust coefficients: $C_{t}=0.61$ (a), $C_{t}=0.86(\mathrm{~b})$ and $C_{t}=0.94$ (c).

\section{Conclusions}

A new turbine model which can be used as a component of a framework for simulating entire floating tidal systems has been presented. Analysis of the new model in steady-state conditions showed that the prediction of the free stream velocity could be replicated to within a $2 \%$ accuracy relative to theoretical solutions, and this could be further reduced by tuning the width parameter and mesh resolution. However, the key aspects of the model were defined by the requirement to use the model for simulation of entire floating tidal systems: the model has been shown to be insensitive to flow velocity, performs well in any alignment with the mesh, and is capable of predicting the free stream velocity while moving through the mesh under both linear and angular velocity. These properties are crucial when simulating floating tidal systems, since such systems will be required to survive in complex, non-linear environments driven by strong wave-current interactions, requiring the turbine methodology to be robust during changing flow velocity. Furthermore, the system will be capable of moving in 6 degrees of freedom, and hence, the turbine will generally be arbitrarily aligned with a mesh, and must be able to accurately predict the free stream velocity under both linear and angular movement.

Following the success of the turbine model presented in this work, future research will focus on the development of a new coupled framework for simulating entire floating tidal systems, including integration of the present model. 


\section{Acknowledgements}

The authors acknowledge that, this work was funded as part of Innovate UK Project 102217 through the Energy Catalyst Early-stage Round 3. The code presented here is available through the Collaborative Computational Project in Wave Structure Interaction (CCP-WSI) [EP/M022382/1], which aims to bring together the community of researchers, data, code and expertise within the area of wave structure interaction (WSI) and has the objective of building an opensource, community-driven numerical wave tank (NWT) facility and capacity for high quality research in an area of high importance (https://www.ccp-wsi. ac.uk).

\section{References}

[1] Abolghasemi, M., Piggott, M., Spinneken, J., Viré, A., C.J., C., Crammond, S., 2016. Simulating tidal turbines with multi-scale mesh optimisation techniques. Journal of Fluids and Structures 66, 69-90.

[2] Ammara, I., Leclerc, C., Masson, C., 2002. A viscous three-dimensional method for aerodynamic analysis of wind farms. J. Sol. Energ. Eng. 124, $345-356$.

[3] Aubrun, S., Loyer, S., Hancock, P., Hayden, P., 2013. Wind turbine wake properties: Comparison between a non-rotating simplified wind turbine model and a rotating model. J. Wind Eng. Ind. Aerodyn. 120, 1-8.

[4] Batten, W., Harrison, M., Bahaj, A., 2013. Accuracy of the actuator discRANS approach for predicting the performance and wake of tidal turbines. Philosophical Transactions of the Royal Society A 371.

[5] Blackmore, T., Batten, W., Bahaj, A., 2014. Influence of turbulence on the wake of a marine current turbine simulator. Proc. R. Soc. A 470, 17.

[6] Burton, T., Jenkins, N., Sharpe, D., Bossanyi, E., 2001. Wind Energy Handbook. 2 ed., John Wiley \& Sons, Ltd.

[7] Castellani, F., Vignaroli, A., 2013. An application of the actuator disc model for wind turbine wakes calculations. Appl. Energ. 101, 432-440.

[8] Churchfield, M., Li, Y., Moriarty, P., 2013. A large-eddy simulation study of wake propagation and power production in an array of tidal-current turbines. Phil. Trans. R. Soc A 371.

[9] Greaves, D., Borthwick, A., 1999. Hierarchical tree-based finite element mesh generation. Int. J. Numer. Meth. Eng. 45, 447-471.

[10] Greaves, D.M., 2004. A quadtree adaptive method for simulating fluid flows with moving interfaces. Journal of Computational Physics 194, 35-56. 
[11] Hansen, M., 2000. Aerodynamics of wind turbines. 1 ed., James \& James (Science Publishers) Ltd.

[12] Harrison, M., Batten, W., Bahaj, A., 2010a. A blade element actuator disc approach applied to tidal stream turbines, in: Proceedings of OCEANS, IEEE, pp. 1-8.

[13] Harrison, M., Batten, W., Bahaj, A., Myers, L., 2009. A comparison between CFD simulations and experiments for predicting the far wake of horizontal axis tidal turbines, in: Proceedings of the 8th European Wave and Tidal Energy Conference, Uppsala, Sweden. pp. 566-575.

[14] Harrison, M., Batten, W., Myers, L., Bahaj, A., 2010b. Comparison between CFD simulations and experiments for predicting the far wake of horizontal axis tidal turbines. IET Renewable Power Generation 4, 613627.

[15] Holst, M., Dahlhaug, O., Faudot, C., 2014. CFD analysis of wave-induced loads on tidal turbine blades. IEEE J. Ocean. Eng. 40, 506-521.

[16] Howard, R., Pereira, J., 2006. A study of wind turbine power generation and turbine/tower interaction using large eddy simulation. Wind Struct. 9, 95-108.

[17] Jacobsen, N.G., Fuhrman, D.R., Fredsøe, J., 2012. A wave generation toolbox for the open-source CFD library: OpenFOAM ${ }^{\circledR}$. International Journal for Numerical Methods in Fluids 70, 1073-1088.

[18] Johnson, B., Francis, J., Howe, J., Whitty, J., 2014. Computational actuator disc models for wind and tidal applications. J. Renew. Energ. 2014, 10 .

[19] Lawson, M., Li, Y., Sale, D., 2011. Development and verification of a computational fluid dynamics model of a horizontal-axis tidal current turbine, in: Proceedings of the 30th International Conference on Ocean, Offshore, and Arctic Engineering, Rotterdam, The Netherlands. pp. 1-10.

[20] Leclerc, C., Masson, C., 2004. Toward blade-tip vortex simulation with an actuator-lifting surface model. AIAA Paper 2004-0667, 9.

[21] Li, Y., Paik, K.J., Xing, T., Carrica, P., 2012. Dynamic overset CFD simulations of wind turbine aerodynamics. Renew. Energ. 37, 285-298.

[22] Lignarolo, L., Ragni, D., Ferreira, C., van Bassel, G., 2016. Experimental comparison of a wind-turbine and of an actuator-disc near wake. J. Renew. Sustain. Energ. 8, 26.

[23] Madsen, H., Bak, C., Døssing, M., Mikkelsen, R., Øye, S., 2010. Validation and modification of the Blade Element Momentum theory based on comparisons with actuator disc simulations. Wind Energ. 13, 373-389. 
[24] Malki, R., Williams, A., Croft, T., Togneri, M., Masters, I., 2013. A coupled blade element momentum - computational fluid dynamics model for evaluating tidal stream turbine performance. Appl. Math. Model. 37, $3006-3020$.

[25] Martínez, L., Leonardi, S., Churchfield, M., Moriarty, P., 2012. A comparison of actuator disk and actuator line wind turbine models and best practices for their use, in: 50th AIAA Aerospace Sciences Meeting, p. 13.

[26] Martínez-Tossas, L., Churchfield, M., Leonardi, S., 2015. Large eddy simulations of the flow past wind turbines: actuator line and disk modeling. Wind Energy 18, 1047-1060.

[27] Masters, I., Chapman, J., Willis, M., Orme, J., 2011. A robust blade element momentum theory model for tidal stream turbines including tip and hub loss corrections. J. Mar. Eng. Techno. 10, 25-35.

[28] Menter, F.R., 1994. Two-equation eddy-viscosity turbulence models for engineering applications. AIAA Journal 32, 1598-1605.

[29] Mikkelsen, R., 2003. Actuator disc methods applied to wind turbines. Ph.D. thesis. Technical University of Denmark.

[30] Moriarty, P., Hansen, A., 2005. AeroDyn Theory Manual. Technical Report NREL/TP-500-36881. NREL.

[31] Myers, L., Bahaj, A., 2010. Experimental analysis of the flow field around horizontal axis tidal turbines by use of scale mesh disk rotor simulators. Ocean Engineering 37, 218-227.

[32] Naderi, S., Parvanehmasiha, S., Torabi, F., 2018. Modeling of horizontal axis wind turbine wakes in Horns Rev offshore wind farm using an improved actuator disc model coupled with computational fluid dynamic. Energy Conversion and Management 171, 953-968.

[33] Naderi, S., Torabi, F., 2017. Numerical investigation of wake behind a HAWT using modified actuator disc method. Energy Conversion and Management 148, 1346-1357.

[34] Nguyen, V., Guillou, S., Thiébot, J., Cruz, A., 2016. Modelling turbulence with an Actuator Disk representing a tidal turbine. Renewable Energy 97, 625-635.

[35] Pintar, M., Kolios, A., 2013. Design of a novel experimental facility for testing of tidal arrays. Energies 6, 4117-4133.

[36] Rajagopalan, R., Rickerl, T., 1990. Aerodynamic interference of vertical axis wind turbines. J. Propul. Power 6, 645-653. 
[37] Rusche, H., 2002. Computational fluid dynamics of dispersed two-phase flows at high phase fractions. Ph.D. thesis. Imperial College of Science, Technology \& Medicine.

[38] Sanderse, B., van der Pijl, S., Korne, B., 2011. Review of computational fluid dynamics for wind turbine wake aerodynamics. Wind Energy 14, 799-819.

[39] Shen, W., Zhang, J., Søresen, J., 2005. Tip loss correction for actuator/Navier-Stokes computations. J. Sol. Energ. 127, 5.

[40] Shen, W., Zhang, J., Søresen, J., 2009. The actuator surface model: A new Navier-Stokes based model for rotor computations. J. Sol. Energ. 131, 9.

[41] Sørensen, J., Mikkelsen, R., Henningson, D., Ivanell, S., Sarmast, S. amd Andersen, S., 2015. Simulation of wind turbine wakes using the actuator line technique. Phil. Trans. R. Soc. A 373, 16.

[42] Sørensen, J., Shen, W., 2002. Numerical modeling of wind turbine wakes. J. Fluid. Eng. 124, 393-399.

[43] Sørensen, N., Michelsen, J., Schreck, S., 2002. Navier-Stokes predictions of the NREL phase VI rotor in the NASA Ames $80 \mathrm{ft} \times 120 \mathrm{ft}$ wind tunnel. Wind Energ. 5, 151-169.

[44] Svenning, E., 2010. Implementation of an actuator disk in OpenFOAM. Technical Report. Chalmers University of Technology.

[45] Viré, A., Xiang, J., Piggott, M., Cotter, C., Pain, C., 2013. Towards the fully-coupled numerical modelling of floating wind turbines. Energy Procedia 35, 43-51.

[46] Wang, Q., Zhou, H., Wan, D., 2012. Numerical simulation of wind turbine blade-tower interaction. J. Mar. Sci. Appli. 11, 321-327.

[47] Watters, C., Masson, C., 2010. Modeling of lifting-device aerodynamics using the actuator surface concept. Int. J. Numer. Meth. Fluids 62, 12641298 .

[48] Weller, H., Greenshields, C., Bainbridge, W., Janssens, M., Santos, B., 2017. OpenFOAM 4.1. URL: https://openfoam.org/version/4-1/.

[49] Wilson, J., Davis, C., Venayagamoorthy, S., Heyliger, P., 2015. Comparisons of horizontal-axis wind turbine wake interaction models. Journal of Solar Energy Engineering 137, 8.

[50] Zahle, F., Søresen, N., Johansen, J., 2009. Wind turbine rotor-tower interaction using an incompressible overset grid method. Wind Energ. 12, 594-619. 